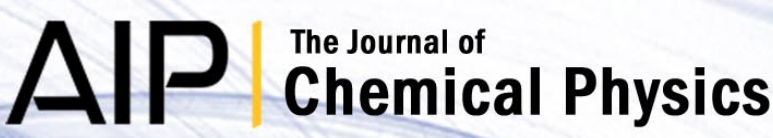

Semiclassical perturbation theory for diffraction in heavy atom surface scattering

Salvador Miret-Artés, Shauli Daon, and Eli Pollak

Citation: J. Chem. Phys. 136, 204707 (2012); doi: 10.1063/1.4722339

View online: http://dx.doi.org/10.1063/1.4722339

View Table of Contents: http://jcp.aip.org/resource/1/JCPSA6/v136/i20

Published by the American Institute of Physics.

\section{Additional information on J. Chem. Phys.}

Journal Homepage: http://jcp.aip.org/

Journal Information: http://jcp.aip.org/about/about_the_journal

Top downloads: http://jcp.aip.org/features/most_downloaded

Information for Authors: http://jcp.aip.org/authors

\section{ADVERTISEMENT}
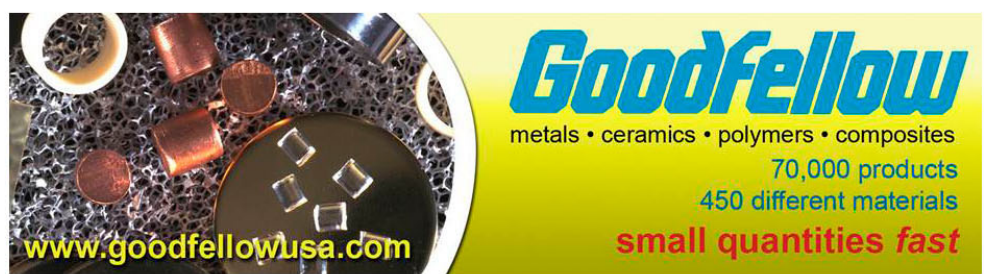


\title{
Semiclassical perturbation theory for diffraction in heavy atom surface scattering
}

\author{
Salvador Miret-Artés, ${ }^{1, a)}$ Shauli Daon, ${ }^{2}$ and Eli Pollak ${ }^{2, b)}$ \\ ${ }^{1}$ Instituto de Física Fundamental, Consejo Superior de Investigaciones Científicas, \\ Serrano 123, 28006 Madrid, Spain \\ ${ }^{2}$ Chemical Physics Department, Weizmann Institute of Science, 76100 Rehovoth, Israel
}

(Received 26 March 2012; accepted 11 May 2012; published online 31 May 2012)

\begin{abstract}
The semiclassical perturbation theory formalism of Hubbard and Miller [J. Chem. Phys. 78, 1801 (1983)] for atom surface scattering is used to explore the possibility of observation of heavy atom diffractive scattering. In the limit of vanishing $\hbar$ the semiclassical theory is shown to reduce to the classical perturbation theory. The quantum diffraction pattern is sensitive to the characteristics of the beam of incoming particles. Necessary conditions for observation of quantum diffraction are derived for the angular width of the incoming beam. An analytic expression for the angular distribution as a function of the angular and momentum variance of the incoming beam is obtained. We show both analytically and through some numerical results that increasing the angular width of the incident beam leads to decoherence of the quantum diffraction peaks and one approaches the classical limit. However, the incoherence of the beam in the parallel direction does not destroy the diffraction pattern. We consider the specific example of Ar atoms scattered from a rigid $\mathrm{LiF}(100)$ surface. (C) 2012 American Institute of Physics. [http://dx.doi.org/10.1063/1.4722339]
\end{abstract}

\section{INTRODUCTION}

A variety of experiments have been carried out during the past few decades in which one observed quantum diffraction effects in the scattering of heavy atoms from surfaces. The seminal diffraction peaks observed by Estermann and Stern in their observations of scattering of $\mathrm{He}$ atoms from a $\mathrm{LiF}$ surface have led to further studies of scattering from surfaces. ${ }^{2}$ The observation of diffraction in atoms heavier than $\mathrm{He}$ was discovered for $\mathrm{Ne}$ when scattered from a LiF surface by Williams ${ }^{3}$ and Boato et al. ${ }^{4}$ Diffraction in Ne scattering on metallic surfaces was then observed by Rieder and Stocker. ${ }^{5}$ Schweizer et al. ${ }^{6,7}$ were the first to measure diffractive scattering of $\mathrm{Ar}$ atoms from a hydrogen covered tungsten surface. Very sharp diffraction features were measured for the scattering of $\mathrm{Ne}$, Ar, and $\mathrm{Kr}$ atoms as well as diatomics such as, $\mathrm{D}_{2}$ and $\mathrm{N}_{2}$ from a $\mathrm{Cu}(111)$ surface by Andersson et al. ${ }^{8,9}$ Typically though the quantum effects were observed for low energy beams, presumably to assure that the deBroglie wavelength is not too small as compared with the lattice length of the surface. ${ }^{6}$

In more recent years, it was realized that quantum diffraction effects may be observed even at high beam energies ${ }^{10,11}$ and very large masses. ${ }^{12-14} \mathrm{~A}$ critical property which determines the quantum nature of the scattering event is the extent of collimation of the incident beam. A well collimated beam will have a large coherence wavelength in the direction perpendicular to the motion, so that diffraction may still be observed. This is why diffraction was observed for very fast atoms impinging on a surface at grazing conditions. ${ }^{10}$

\footnotetext{
a)Electronic mail: s.miret@iff.csic.es.

b) Electronic mail: eli.pollak@weizmann.ac.il.
}

It was also found in the scattering of very heavy molecules through gratings. Moix and Pollak ${ }^{15}$ have recently used model wavepacket propagation methods to demonstrate how the collimation of a beam of incident Ar atoms may affect the measured angular distribution. When the collimation was strong, such that the perpendicular coherence wavelength was longer than the lattice length, one found that the angular distribution was dominated by diffraction peaks. When the angular resolution of the beam was poor, the angular distribution reverted to its classical limit.

In this present paper, we will adapt the semiclassical perturbation (SCP) theory of Hubbard and Miller ${ }^{1,16}$ to study how collimation affects the measured angular distribution. In Sec. II, the SCP formalism will be adapted for the final momentum and angular distributions. We show that the SCP theory reduces to the classical perturbation theory we have been using in recent years to analyze angular distributions of heavy atom surface scattering. ${ }^{17-21}$ We then account for the initial beam conditions by introducing an appropriate Gaussian averaging over initial conditions. The classical distribution is obtained in the limit of a large angular width of the beam as compared to the angular distance between successive diffraction peaks. The formalism is then applied in Sec. III, using a model potential whose parameters have been previously fitted ${ }^{18,21}$ for the observed scattering of $\mathrm{Ar}$ from a $\mathrm{LiF}$ surface. ${ }^{22}$ We demonstrate that the SCP based theory may be used to estimate the effect of collimation of the incident beam on the measured angular distribution. Depending on the extent of angular collimation one can move smoothly from a fully quantum diffraction regime, to the fully incoherent classical regime for the angular distribution. We also find that the incoherence of the beam in the direction of propagation does not destroy the quantum diffraction pattern. We end with a discussion in Sec. IV. 


\section{SEMICLASSICAL PERTURBATION THEORY OF ATOM SURFACE SCATTERING}

\section{A. Classical perturbation theory}

We will model the dynamics in terms of two degrees of freedom, a vertical coordinate $z$ (with conjugate momentum $p_{z}$ ) describing the distance of the atom (whose mass is $M$ ) from the surface, and a horizontal coordinate $x$ (with conjugate momentum $p_{x}$ ) for motion parallel to the surface. The theory presented is readily generalized to include an additional $(y)$ coordinate for motion parallel to the surface, but usually, one measures the in-plane angular distribution so that the limitation to a description with only two degrees of freedom is reasonable and illuminates the results. The usage of a two dimensional model is justified whenever the corrugation may be approximated as an independent sum of corrugations in the two orthogonal horizontal directions. We also note that this is the usual assumption made in many experimental ${ }^{4}$ and theoretical papers (for a review see, for example, Ref. 21). The Hamiltonian for the scattering of an atom of mass $M$ from a corrugated surface is then written as ${ }^{17-19}$

$$
H=\frac{p_{x}^{2}+p_{z}^{2}}{2 M}+\bar{V}(z)+\bar{V}^{\prime}(z) h(x),
$$

where the interaction potential is split into two terms. $\bar{V}(z)$ is the zeroth order term (for analytic purposes it is taken as a Morse potential) and the perturbation term is given by the product of the first derivative of the vertical potential times the periodic corrugation function $h(x)$, with period (lattice length) $l$ assumed for the surface.

The SCP is based on a classical perturbation theory, in which the small parameter is the height $h$ of the corrugation potential $h(x)$. The unperturbed motion in the vertical direction is governed by the vertical Hamiltonian

$$
H_{z}=\frac{p_{z}^{2}}{2 M}+\bar{V}(z)
$$

while to zeroth order, the parallel momentum is conserved. Using a time scale in which the particle reaches the turning point of the vertical potential $\bar{V}(z)$ at time $t=0$ one has from the solution of the classical equation of motion for the horizontal momentum that the first order change due to the collision at the horizontal point of impact $x_{0}$ is given by

$$
\delta p_{x}\left(x_{0}\right)=-\int_{-\infty}^{\infty} d t \bar{V}^{\prime}\left(z_{t}\right) h^{\prime}\left[x_{0}+\frac{p_{x_{i}}}{M} t\right],
$$

where $z_{t}$ is the vertical motion as obtained from the zeroth order Hamiltonian (2.2) and $p_{x_{i}}$ is the incident (positive) momentum of the particle in the horizontal direction. The change in the vertical momentum is readily obtained from energy conservation. Denoting the initial vertical momentum as $p_{z_{i}}$ (assumed negative) and retaining only linear terms in $\delta p_{x}$, one readily finds that the vertical momentum change is

$$
\delta p_{z}\left(x_{0}\right)=\frac{\delta p_{x}\left(x_{0}\right) p_{x_{i}}}{p_{z_{i}}} .
$$

The probability for observing the particle with final momenta $p_{x_{f}}$ and $p_{z_{f}}$ is then obtained by averaging over the im- pact points,

$$
\begin{aligned}
P\left(p_{x_{f}}, p_{z_{f}}\right)= & \frac{1}{l} \int_{0}^{l} d x_{0} \delta\left(p_{x_{f}}-p_{x_{i}}-\delta p_{x}\left(x_{0}\right)\right) \\
& \times \delta\left(p_{z_{f}}+p_{z_{i}}-\delta p_{z}\left(\delta p_{z}\left(x_{0}\right)\right)\right)
\end{aligned}
$$

where $\delta(x)$ denotes the Dirac "delta" function. The (negative) angle of incidence $\theta_{i}$ and the final scattering angle $\theta_{f}$ relative to the normal to the surface are by definition

$$
\theta_{j}=\tan ^{-1}\left(\frac{p_{x_{j}}}{p_{z_{j}}}\right), \quad j=i, f .
$$

The final classical angular distribution is then given in terms of the final momentum distribution as,

$$
\begin{aligned}
P\left(\theta_{f}\right)= & \int_{-\infty}^{\infty} d p_{x_{f}} \int_{0}^{\infty} d p_{z_{f}} \delta\left(\theta_{f}-\tan ^{-1}\left(\frac{p_{x_{f}}}{p_{z_{f}}}\right)\right) P\left(p_{x_{f}}, p_{z_{f}}\right) \\
= & \frac{1}{l \cos ^{2} \theta_{f}} \int_{0}^{l} d x_{0} \delta\left(\tan \theta_{f}+\tan \theta_{i}\left(1+\frac{\delta p_{x}\left(x_{0}\right)}{p_{x_{i}} \cos ^{2} \theta_{i}}\right)\right) \\
& \times H\left(1-\frac{\delta p_{x}\left(x_{0}\right) \tan ^{2} \theta_{i}}{p_{x_{i}}}\right)
\end{aligned}
$$

where $H(x)$ is the Heaviside function; we used the identity

$$
\delta\left(\theta-\tan ^{-1}(\alpha)\right)=\cos ^{-2} \theta \delta(\alpha-\tan \theta)
$$

and to remain consistent with the first order perturbation theory we have retained only terms which are up to linear in the horizontal momentum change $\delta p_{x}\left(x_{0}\right)$.

\section{B. Semiclassical perturbation theory}

Following Hubbard and Miller, ${ }^{1,16,23}$ the S-matrix element or amplitude for scattering to a final diffraction channel characterized by the quantum number $n$ from the specular channel 0 is given within the SCP theory approximation by

$$
S_{n 0}=\frac{1}{l} \int_{0}^{l} d x_{0} e^{-i \Delta k_{x} x_{0}} e^{2 i \eta\left(x_{0}\right)},
$$

where the phase shift $\eta$ is given by

$$
2 \eta\left(x_{0}\right)=-\frac{1}{\hbar} \int_{-\infty}^{+\infty} d t V\left(x_{t}, z_{t}\right),
$$

where $x_{t}$ and $z_{t}$ denote the zeroth order trajectories, as in the classical perturbation theory. The diffraction order number $n$ is defined through the change of the horizontal component of the wavevector $\Delta k_{x}=k_{x_{f}}-k_{x_{i}}$ and guided by Bragg's law as,

$$
n=\frac{l \Delta k_{x}}{2 \pi}=\frac{l \delta p_{x}}{2 \pi \hbar}
$$

but here the horizontal momentum change is no longer a function of the impact parameter $x_{0}$ as in the classical case (Eq. (2.3)). Equation (2.9) can be written in a more standard form as

$$
S_{n 0}=(2 \pi)^{-1} \int_{0}^{2 \pi} d q_{x} e^{-i n q_{x}} e^{2 i \eta\left(q_{x}\right)}
$$

in terms of the angle variable

$$
q_{x}=2 \pi x_{0} / l,
$$


which is conjugate to the diffraction order number $n$. The diffraction probability is given by

$$
P_{n 0}=\left|S_{n 0}\right|^{2} \text {. }
$$

Hubbard and Miller have shown that the SCP estimate of the transition probability is indeed a very good approximation to the exact quantum probability. ${ }^{1}$

In quantum mechanics, the diffraction order number is necessarily an integer so that the final distribution of momenta is

$$
\begin{aligned}
P\left(p_{x_{f}}, p_{z_{f}}\right)= & \sum_{n=-\infty}^{\infty} \delta\left(p_{x_{f}}-p_{x_{i}}-\frac{2 \pi \hbar n}{l}\right) \\
& \times \delta\left(p_{z_{f}}+p_{z_{i}}-\delta p_{z}\left(\delta p_{x}\right)\right)\left|S_{n 0}\right|^{2} .
\end{aligned}
$$

The change in the vertical momentum is obtained as in the classical case through the conservation of energy (see Eq. (2.4)). The SCP expression for the angular distribution is obtained as in the first equality in Eq. (2.7).

\section{The classical limit of the SCP expression}

It is of interest to show that the semiclassical expression for the momentum distribution reduces in the limit that $\hbar \rightarrow 0$ to the corresponding classical expression. If in Eq. (2.15) the sum over $n$ going from $-\infty$ to $+\infty$ is replaced by an integral over the variable $\hbar n$ then the final momentum distribution is

$$
\begin{aligned}
P\left(p_{x_{f}}, p_{z_{f}}\right)= & \frac{l}{\hbar(2 \pi)^{3}} \int_{0}^{2 \pi} d q_{x} \int_{0}^{2 \pi} d q_{x}^{\prime} \delta\left(p_{z_{f}}+p_{z_{i}}-\delta p_{z}\left(\delta p_{x}\right)\right) \\
& \cdot \exp \left(-i \frac{l \delta p_{x}}{2 \pi \hbar}\left(q_{x}-q_{x}^{\prime}\right)+2 i\left[\eta\left(q_{x}\right)-\eta\left(q_{x}^{\prime}\right)\right]\right) .
\end{aligned}
$$

Using the sum and difference variables

$$
Q=\frac{q_{x}+q_{x}^{\prime}}{2}, \quad \Delta Q=q_{x}-q_{x}^{\prime}
$$

and linearizing with respect to the difference variable,

$$
\eta\left(q_{x}\right)-\eta\left(q_{x}^{\prime}\right) \simeq \eta^{\prime}(Q) \Delta Q
$$

we readily find that in the limit that $\hbar \rightarrow 0$, the momentum distribution becomes (with $\Delta Q^{\prime}=\Delta Q / \hbar$ )

$$
\begin{aligned}
P\left(p_{x_{f}}, p_{z_{f}}\right) \simeq & \frac{l}{(2 \pi)^{3}} \int_{0}^{2 \pi} d Q \int_{-Q / \hbar}^{Q / \hbar} d \Delta Q^{\prime} \delta\left(p_{z_{f}}+p_{z_{i}}-\delta p_{z}\left(\delta p_{x}\right)\right) \\
& \times \exp \left(-i \hbar n \Delta Q^{\prime}+2 i \hbar \eta^{\prime}(Q) \Delta Q^{\prime}\right) \\
= & \frac{1}{l} \int_{0}^{l} d x_{0} \delta\left(p_{z_{f}}+p_{z_{i}}-\delta p_{z}\left(\delta p_{x}\right)\right) \\
& \times \delta\left(\delta p_{x}-\frac{4 \pi}{l} \hbar \eta^{\prime}\left(\frac{2 \pi x_{0}}{l}\right)\right)
\end{aligned}
$$

Using the definition for the phase shift as in Eq. (2.10) one readily finds that this is indeed the classical perturbation theory expression given in Eq. (2.5).

\section{The SCP theory for a sine corrugation function}

If we now assume a sine corrugation function such that

$$
h(x)=h \sin (2 \pi x / l)
$$

then

$$
2 \eta\left(q_{x}\right)=2 \eta_{0}-\frac{h}{\hbar} \int_{-\infty}^{+\infty} d t \bar{V}^{\prime}\left(z_{t}\right) \sin \left(q_{x}+\omega_{x} t\right),
$$

where $2 \eta_{0}=-(1 / \hbar) \int_{-\infty}^{+\infty} d t \bar{V}\left(z_{t}\right)$ is the constant phase shift due to the unperturbed potential $\bar{V}(z)$ and the frequency along the horizontal direction due to the rectilinear motion is $\omega_{x}$ $=2 \pi p_{x_{i}} /(M l)$. By noting that $\bar{V}^{\prime}\left(z_{t}\right)$ is an even function in time and inserting Eq. (2.21) into Eq. (2.9) one finds that ${ }^{1}$

$$
S_{n 0}=\exp \left(2 i \eta_{0}\right) J_{n}\left(\frac{A_{x}}{\hbar}\right),
$$

where $J_{n}(y)$ is the $n$th order Bessel function,

$$
A_{x}=h \int_{-\infty}^{+\infty} d t \bar{V}^{\prime}\left(z_{t}\right) \cos \omega_{x} t
$$

and following from Eq. (2.14) the diffraction probability is

$$
P_{n 0}=J_{n}^{2}\left(\frac{A_{x}}{\hbar}\right)
$$

The action variable $A_{x}$ is related to the rainbow shift angle function $K$ which arises in the classical perturbation theory $^{17-19}$

$$
K\left(p_{x_{i}}, p_{z_{i}}\right)=\frac{2 \pi}{l p_{z_{i}}} A_{x}
$$

\section{E. Averaging over initial velocities}

Typically the incident beam has a Gaussian distribution of velocities centered about some average values. We may assume that this distribution takes the form

$$
P\left(p_{\|}, p_{\perp} ; \bar{p}_{\|}\right)=\frac{1}{\pi \sigma_{\|} \sigma_{\perp}} \exp \left(-\frac{\left(p_{\|}-\bar{p}_{\|}\right)^{2}}{\sigma_{\|}^{2}}-\frac{p_{\perp}^{2}}{\sigma_{\perp}^{2}}\right),
$$

where $p_{\|}$is the (negative) incident momentum in the direction of the average propagation of the beam while $p_{\perp}$ is the incident momentum perpendicular to the average direction of propagation. The average incident (negative) scattering angle $\bar{\theta}_{i}$ is by definition the angle between the average (negative) parallel momentum $\bar{p}_{\|}$and the $\mathrm{z}$ axis. We then have that the parallel and perpendicular momenta may be expressed in terms of the vertical and horizontal incident momenta as,

$$
\begin{aligned}
& p_{\|}=p_{z_{i}} \cos \left(\bar{\theta}_{i}\right)+p_{x_{i}} \sin \left(\bar{\theta}_{i}\right)=-p_{i} \cos \left(\theta_{i}-\bar{\theta}_{i}\right), \\
& p_{\perp}=-p_{z_{i}} \sin \left(\bar{\theta}_{i}\right)+p_{x_{i}} \cos \left(\bar{\theta}_{i}\right)=p_{i} \sin \left(\theta_{i}-\bar{\theta}_{i}\right),
\end{aligned}
$$

with

$$
p_{i}^{2}=p_{x_{i}}^{2}+p_{z_{i}}^{2}=\sqrt{2 M E_{i}}
$$


Inserting these expressions into the incident momentum distribution (Eq. (2.26)) implies that it may be written as

$$
\begin{aligned}
P\left(p_{\|}, p_{\perp} ; \bar{p}_{\|}, \bar{\theta}_{i}\right)= & \frac{1}{\pi \sigma_{\|} \sigma_{\perp}} \exp \left(-\frac{\left(p_{i} \cos \left(\theta_{i}-\bar{\theta}_{i}\right)+\bar{p}_{\|}\right)^{2}}{\sigma_{\|}^{2}}\right. \\
& \left.-\frac{p_{i}^{2} \sin ^{2}\left(\theta_{i}-\bar{\theta}_{i}\right)}{\sigma_{\perp}^{2}}\right) .
\end{aligned}
$$

In the limit that we have "good" collimation in the perpendicular direction we can approximate the distribution to be

$$
\begin{aligned}
& P\left(p_{\|}, p_{\perp} ; \bar{p}_{\|}, \bar{\theta}_{i}\right) \\
& \quad \simeq \frac{1}{\pi \sigma_{\|} \sigma_{\perp}} \exp \left(-\frac{\left(p_{i}+\bar{p}_{\|}\right)^{2}}{\sigma_{\|}^{2}}-\frac{p_{i}^{2}\left(\theta_{i}-\bar{\theta}_{i}\right)^{2}}{\sigma_{\perp}^{2}}\right) .
\end{aligned}
$$

Using the notation

$$
B_{n}\left(\theta_{i}\right)=\frac{2 \pi \hbar n}{l p_{i} \cos \theta_{i}},
$$

and replacing $B_{n}\left(\theta_{i}\right)$ with its value at the maximum, $B_{n}\left(\bar{\theta}_{i}\right)$ and similarly for $\left|S_{n 0}\right|^{2}$ we find that the final angular distribution is

$$
\begin{aligned}
P\left(\theta ; \bar{p}_{\|}, \bar{\theta}_{i}\right) \simeq & \sum_{n=-\infty}^{\infty} \int_{0}^{\infty} d p_{i} p_{i} \frac{\left|S_{n 0}\left(p_{i}, \bar{\theta}_{i}\right)\right|^{2}}{\pi \sigma_{\|} \sigma_{\perp}} \cdot \exp \left(-\frac{\left(p_{i}+\bar{p}_{\|}\right)^{2}}{\sigma_{\|}^{2}}\right. \\
& \left.-\frac{p_{i}^{2}\left(\tan ^{-1}\left[B_{n}\left(\bar{\theta}_{i}\right)\right]-\theta-\bar{\theta}_{i}\right)^{2}}{\sigma_{\perp}^{2}}\right)
\end{aligned}
$$

and the remaining integral over the initial momentum has to be carried out numerically.

This result is already instructive. Note that the uncertainty in the parallel momentum only serves to average out the magnitude of the separate diffraction probabilities, but it does not affect the diffraction pattern itself. Each separate diffraction peak is smeared only through the uncertainty in the perpendicular momentum, as expressed by the angular width $\sigma_{\perp} /\left(\sqrt{2} p_{i}\right)$. This demonstrates that sufficient collimation will reveal the underlying diffraction structure of the angular momentum distribution. It also provides a quick estimate for the collimation needed to expose the diffraction pattern. The angular distance between successive diffraction peaks is

$$
\Delta \theta_{n}=\tan ^{-1}\left[B_{n+1}\left(\bar{\theta}_{i}\right)\right]-\tan ^{-1}\left[B_{n}\left(\bar{\theta}_{i}\right)\right] .
$$

Thus, the condition for the observation of the $n$th diffraction peak is

$$
\frac{\sigma_{\perp}}{p_{i}} \ll \Delta \theta_{n} .
$$

We note that the angle difference between diffraction peaks is not linear in the diffraction number. As the diffraction number increases, the angle difference decreases and a better angular resolution is needed to resolve the peak. As is well-known, the angle difference decreases with increasing momentum of the projectile and with increasing mass, as may be also discerned from Eqs. (2.32) and (2.34).

If we further replace $p_{i}$ with its average value $-\bar{p}_{\|}$in the argument of the S-matrix element and the term $\tan ^{-1}\left[B_{n}\left(\bar{\theta}_{i}\right)\right]$ we readily find that the angular distribution reduces to a sum over error functions

$$
\begin{aligned}
P\left(\theta ; \bar{p}_{\|}, \bar{\theta}_{i}\right) \simeq & \sum_{n=-\infty}^{\infty} \frac{\sigma_{\|} \sigma_{\perp}\left|S_{n 0}\left(\bar{p}_{\|}, \bar{\theta}_{i}\right)\right|^{2}}{2 \pi C_{n}^{2}} \\
& \cdot\left(\exp \left(-\frac{\bar{p}_{\|}^{2}}{\sigma_{\|}^{2}}\right)-\frac{\bar{p}_{\|} \sqrt{\pi} \sigma_{\perp}}{\sigma_{\|} C_{n}}\right. \\
& \left.\times \exp \left(-\frac{\bar{p}_{\|}^{2}}{\sigma_{\|}^{2}}\left[\frac{C_{n}^{2}-\sigma_{\perp}^{2}}{C_{n}^{2}}\right]\right) \operatorname{erfc}\left(\frac{\overline{\mathrm{p}}_{\|} \sigma_{\perp}}{\sigma_{\|} C_{\mathrm{n}}}\right)\right)
\end{aligned}
$$

and we used the notation

$$
C_{n}^{2}=\left[\sigma_{\perp}^{2}+\sigma_{\|}^{2}\left(\tan ^{-1}\left[B_{n}\left(\bar{\theta}_{i}\right)\right]-\theta-\bar{\theta}_{i}\right)^{2}\right] .
$$

Equation (2.36) is a central result of this paper. Inserting the SCP approximation for the diffraction probability gives a closed expression for the angular distribution after averaging over the Gaussian profile of the incident beam.

To complete the discussion, it is also worthwhile to write down an expression for the classical angular distribution, when averaged over the profile of the incident beam. For the sine corrugation function (Eq. (2.20)) one finds that

$$
\delta p_{x}\left(x_{0}\right)=-p_{z_{i}} K\left(p_{x_{i}}, p_{z_{i}}\right) \cos \left(\frac{2 \pi x_{0}}{l}\right),
$$

where the rainbow angle shift function $K\left(p_{x_{i}}, p_{z_{i}}\right)$ has been identified in Eq. (2.25). The classical angular distribution becomes,

$$
\begin{aligned}
P\left(\theta_{f}\right)= & \frac{1}{\pi \sqrt{K^{2}\left(p_{x_{i}}, p_{z_{i}}\right) \cos ^{2}\left(\theta_{i}+\theta_{f}\right)-\sin ^{2}\left(\theta_{i}+\theta_{f}\right)}} \\
& \times H\left(K^{2}\left(p_{x_{i}}, p_{z_{i}}\right)-\tan ^{2}\left(\theta_{i}+\theta_{f}\right)\right) .
\end{aligned}
$$

Averaging over the profile of the incident beam is then readily approximated as

$$
\begin{aligned}
\left\langle P\left(\theta_{f}\right)\right\rangle \simeq & \frac{\bar{p}_{\|}}{\pi \sqrt{\pi} \sigma_{\perp}} \int_{-\pi / 2}^{\pi / 2} d u \\
& \times \frac{1}{\sqrt{1+K^{2}\left(p_{x_{i}}, p_{z_{i}}\right)-K^{2}\left(p_{x_{i}}, p_{z_{i}}\right) \sin ^{2} u}} \\
& \cdot \exp \left(-\frac{\bar{p}_{\|}^{2}}{\sigma_{\perp}^{2}}\left[\sin ^{-1}\left(\frac{K\left(p_{x_{i}}, p_{z_{i}}\right)}{\sqrt{1+K^{2}\left(p_{x_{i}}, p_{z_{i}}\right)}} \sin u\right)\right.\right. \\
& \left.\left.-\bar{\theta}_{i}-\theta_{f}\right]^{2}\right) .
\end{aligned}
$$

The averaging over the angular width of the incident beam smooths the classical divergence at the rainbow angles. In the Appendix, we show that this expression is also obtained from the SCP angular distribution (Eq. (2.33)) in the limit that the angular width of the incoming beam is large as compared to the angular distance between successive diffraction peaks. 


\section{APPLICATION TO THE SCATTERING OF Ar ON A LiF SURFACE}

Kondo et al..$^{22}$ have measured an angular distribution for the scattering of $\mathrm{Ar}$ on the $\operatorname{LiF}(100)$ surface. In their experiment, the incidence energy was varied between 300 and $700 \mathrm{meV}$. The angle between the incident beam and the detector was kept fixed at $90^{\circ}$ and the final intensity was measured as a function of the angle of incidence which was varied about $45^{\circ}$. The angular distribution measured in this way is qualitatively similar to the angular distribution measured when the incident angle is fixed at $45^{\circ}$ relative to the vertical axis and the final angle is measured. To simplify then we will discuss here only the final angular distribution measured at the fixed angle of incidence of $45^{\circ}$.

In Refs. 18-21 we used a Morse potential and a sinusoidal corrugation to fit the experimentally measured results. Here, we will use the same model, with a single sine corrugation term with corrugation height $h=0.2$ a.u. The lattice length of the surface is $l=4 \AA$, the Morse potential well depth was chosen as $V_{0}=88 \mathrm{meV}$ and the stiffness parameter $\alpha$ was chosen such that $\alpha l=3$. The various necessary analytic expressions for this model may be found in Refs. 18-21, here for the sake of completeness we just bring the relevant results. The rainbow shift angle is given by the expression

$$
K\left(p_{x_{i}}, p_{z_{i}}\right)=\frac{4 \pi^{2} h}{l} \frac{\bar{\Omega} \cosh (\Phi \bar{\Omega})}{\sinh (\pi \bar{\Omega})},
$$

where the dimensionless parameter

$$
\bar{\Omega}=\frac{2 \pi}{\alpha l}\left|\tan \theta_{i}\right|
$$

and the energy dependent angle $\Phi$ varies from $\pi / 2$ at high incidence energies to $\pi$ at low energies and is defined as,

$$
\cos \Phi=-\sqrt{\frac{V_{0}}{V_{0}+E_{i} \cos ^{2} \theta_{i}}} .
$$

In the experiment the uncertainty in the incidence energy $\left(\Delta E_{i} / E_{i}\right)$ was $\sim 20 \%$ at all energies, implying a momentum uncertainty for the parallel incident momentum of $10 \%$. The experimental paper does not report the angular resolution for the Ar beam, here we use (as also in Ref. 15) a $2^{\circ}$ angular width as our base line. We thus have that at all energies studied experimentally we choose the Gaussian widths of the incident beam as

$$
\frac{\sigma_{\|}}{\sqrt{2} p_{i}}=0.1 x_{\|}
$$

and

$$
\frac{\sigma_{\perp}}{\sqrt{2} p_{i}}=\frac{\pi}{90} x_{\perp}
$$

where it is understood that the experimental conditions are assumed to correspond to the scaling parameters $x_{\|}$and $x_{\perp}$ equaling unity.

In Figure 1 we plot the dependence of the angle difference function as defined in Eq. (2.34) as a function of the quantum number $n$ of the $n$th diffraction peak at two incident energies. At the lower energy $(\mathrm{E}=315 \mathrm{meV})$, the angle difference function is of the order of $1.5^{\circ}$ over the whole region

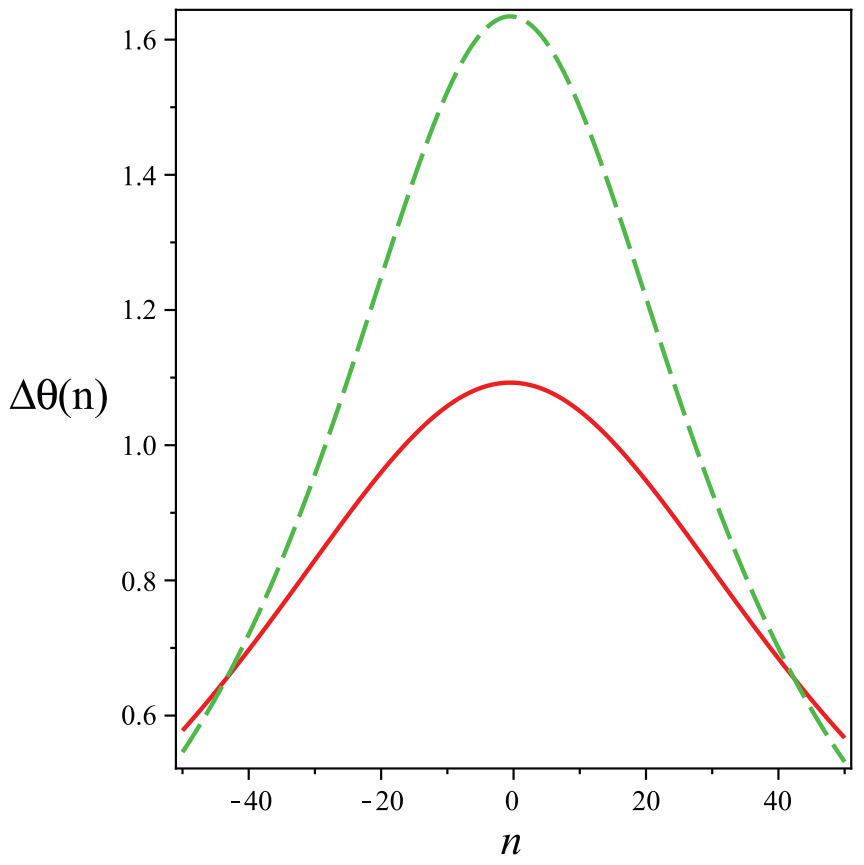

FIG. 1. The angle difference function $\Delta \theta(n)$ (in degrees) needed to resolve individual diffraction peaks is plotted versus the diffraction number $n$ for a model of Ar scattered on a $\operatorname{LiF}(100)$ surface at two energies of incidence. The green (dashed) line is at an energy of $315 \mathrm{meV}$, the red (solid) line is at $E=705 \mathrm{meV}$.

in which one finds measurable scattering amplitude. At the higher energy $(\mathrm{E}=705 \mathrm{meV})$ the angular difference in the experimentally relevant region is $\sim 1^{\circ}$. Clearly a higher angular resolution is needed for the higher energy, as expected from theory and from the observation that as the projectile's energy increases, one may expect it to be closer to the classical limit.

The angular distributions predicted by our model are shown in Figure 2 for the low energy case $(315 \mathrm{meV})$ and in Figure 3 for the high energy case $(705 \mathrm{meV})$. The uncertainty in the parallel momentum is kept fixed in all computations at $10 \%$. In each of the plots, we show four results. The SCP angular distributions as obtained from Eq. (2.36) are plotted for three values of the perpendicular variance parameter $x_{\perp}=1$, $0.25,0.1$ corresponding to angular widths of $2^{\circ}, 0.5^{\circ}$ and $0.1^{\circ}$, respectively. We also show the classical distribution averaged with an angular width of $2^{\circ}$ as obtained from Eq. (2.39).

We first note that the SCP angular distribution is almost identical to the classical one when the angular width is $2^{\circ}$. This is to be expected, since as one can see from Figure 1, the angular difference between successive peaks is at most $1.6^{\circ}$ at this energy. It is also evident that increasing the resolution by an order of magnitude is sufficient for resolving the complete diffraction pattern. At the higher energy, the angular difference between successive peaks is smaller. Although all diffraction peaks are resolved with a width of $0.2^{\circ}$, one notes that the base line is not zero as it is in the lower energy. One would need to decrease the angular width of the incident beam a bit more.

This analysis also explains why typically, one should expect that the diffraction peaks will be less noticeable around the classical rainbow angles. These angles are at the edge of 


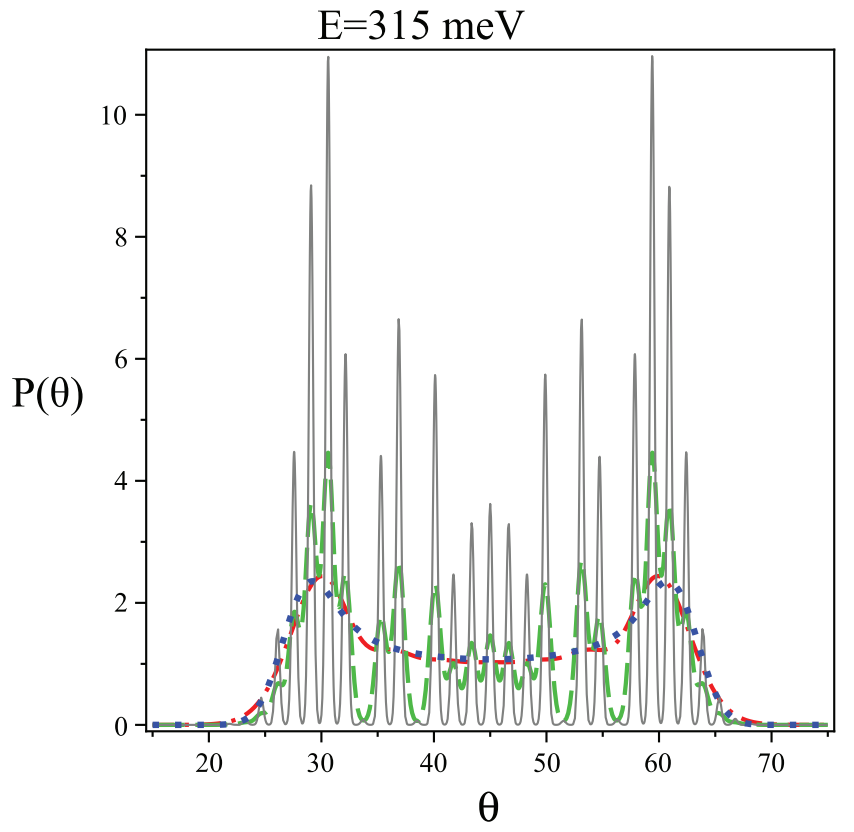

FIG. 2. The SCP angular distribution for the scattering of $\mathrm{Ar}$ on $\operatorname{LiF}(100)$ at $\mathrm{E}=315 \mathrm{meV}$ is plotted for different angular widths of the incident beam. The gray (solid), green (dashed), and red (dashed-dotted) lines correspond to angular widths of $0.2^{\circ}, 0.5^{\circ}$, and $2^{\circ}$, respectively. The blue (dotted) line is the classical distribution obtained with an angular width of $2^{\circ}$. Note the well resolved diffraction peaks for a beam width of $0.2^{\circ}$, while for a beam width of $2^{\circ}$ the $\mathrm{SCP}$ and the classical distributions are almost identical.

the observable distribution, where the diffraction number is higher, so that a higher angular resolution of the incident beam is needed to resolve the separate diffraction peaks appearing under the envelope.

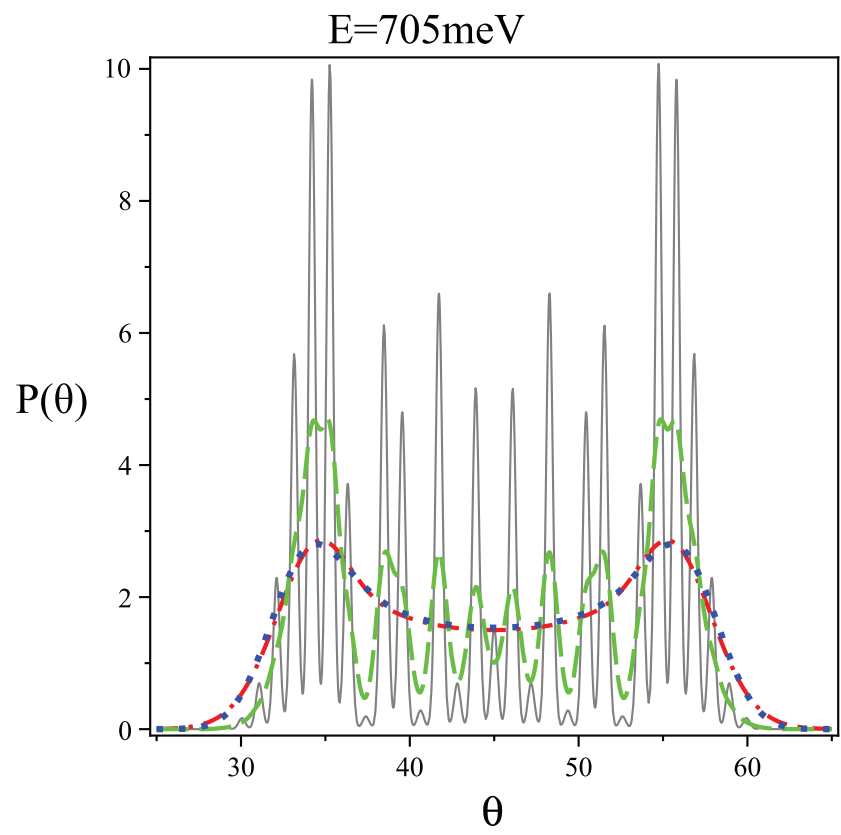

FIG. 3. The SCP angular distribution for the scattering of $\operatorname{Ar}$ on $\operatorname{LiF}(100)$ at $\mathrm{E}=705 \mathrm{meV}$ is plotted for different angular widths of the incident beam. The notation is as in Figure 2. Note that due to the higher energy, the resolution in this figure is somewhat poorer than the resolution with the same beam characteristics as shown in Figure 2 at the energy of $305 \mathrm{meV}$.

\section{DISCUSSION}

In this paper we used the semiclassical perturbation theory of Hubbard and Miller ${ }^{1}$ to show how the angular width of an incident atomic beam may be used to resolve the quantum mechanical diffraction pattern of an atom scattered from a surface. The SCP approximation was shown to reduce in the limit of $\hbar \rightarrow 0$ to the classical perturbation theory expression for the angular distribution used by us extensively in recent years to analyze the experimental data of heavy atom surface scattering experiments. ${ }^{5-9}$ Representing the angular spread of the incoming beam of atoms in terms of a Gaussian distribution, we demonstrated how good collimation of the incident beam may resolve the quantum diffraction peaks even for heavy atom scattering, or high energies. The critical parameter which determines whether one may observe diffraction is the angular width of the incident beam as compared to the angular distance between successive diffraction peaks. This distance, depends inversely on the speed of the projectile and decreases as the square root of the incident particle mass is increased. Both of these aspects are well demonstrated by the experimental results of Boato et al. ${ }^{4} \mathrm{Al}-$ though they use an effusive beam, the strong collimation of the beam to an angular width of less than $0.1^{\circ}$ enabled them to observe diffraction in the scattering of $\mathrm{Ne}$ from $\operatorname{LiF}(001)$. Finally, we showed how the quantum diffraction pattern decoheres and reduces to the classical angular distribution when the angular spread of the incident beam is sufficiently large.

The theory was then applied to a model for the scattering of $\mathrm{Ar}$ on the $\mathrm{LiF}(100)$ surface. Assuming a $2^{\circ}$ angular spread of the incident beam, we showed that this would prevent observation of quantum diffraction, even if the surface is rigid. However, reducing the angular width by a factor of 10 to $0.2^{\circ}$ is sufficient for observing the full diffraction pattern. Of course, the results presented ignore the effects of phonons on the scattering. At room temperature one may assume that the phonon interaction is sufficiently strong so as to destroy the quantum coherence and even with a $0.2^{\circ}$ resolution one would not observe diffraction. However, in this paper we limited ourselves only to consideration of the angular spread, without taking into account additional effects. We do note, that the SCP approach is well adapted also to treat phonons, as may be seen from Ref. 23. An analysis of the effect of phonons on the angular distribution as described within the SCP formalism will be discussed separately. ${ }^{24}$

\section{ACKNOWLEDGMENTS}

We acknowledge the support of the Israel Science Foundation (ISF), the Weizmann-UK Joint Research Program and the Albert Einstein Minerva Center for Theoretical Physics at the Weizmann Institute for their support of this research. S.M.A. also acknowledges the Ministerio de Economia y Competitividad (Spain) in the framework of the project with reference FIS2010-18132. We also acknowledge the support from the European Cooperation in Scientific and Technical Research COST Action MP1006. 


\section{APPENDIX: DERIVING THE CLASSICAL LIMIT WHEN THE ANGULAR SPREAD OF THE INCIDENT BEAM IS LARGE}

In this appendix, we demonstrate analytically, how the quantum angular distribution reduces to the classical, in the limit that the angular width of the incident beam is large compared to the distance between successive diffraction peaks. From Eq. (2.33) we have that the Gaussian averaged quantum angular distribution is well approximated as

$$
\begin{aligned}
P\left(\theta ; \bar{p}_{\|}, \bar{\theta}_{i}\right) & \simeq \sum_{n=-\infty}^{\infty} \frac{1}{\sqrt{\pi} \sigma}\left|S_{n 0}\left(p_{i}, \bar{\theta}_{i}\right)\right|^{2} \\
& \times \exp \left(-\frac{\left(\tan ^{-1}\left[B_{n}\left(\bar{\theta}_{i}\right)\right]-\theta-\bar{\theta}_{i}\right)^{2}}{\sigma^{2}}\right),
\end{aligned}
$$

where we denoted the dimensionless angular width of the incident beam as $\sigma$. In the spirit of the perturbation theory we assume that the angular range of the scattered distribution does not deviate too far from the specular angle, or equivalently that the rainbow angle shift $K \ll 1$ (in the Ar-LiF model considered above, $K \simeq 0.2-0.3$ ). We may then approximate

$$
\tan ^{-1}\left[B_{n}\left(\bar{\theta}_{i}\right)\right] \simeq B_{n}\left(\bar{\theta}_{i}\right) .
$$

The fact that the angular width of the incoming beam is larger than the distance between successive peaks implies that

$$
\sigma \gg B_{n+1}\left(\bar{\theta}_{i}\right)-B_{n}\left(\bar{\theta}_{i}\right)=B_{1}\left(\bar{\theta}_{i}\right)
$$

Using the integral representation of the Bessel functions (see Eqs. (2.9) and (2.22)), replacing the summation over the diffraction quantum number $n$ with an integration over the variable $u=B_{1}\left(\bar{\theta}_{i}\right) n / \sigma$ (which is permitted due to the central assumption of a large angular width as given in Eq. (A3)) allows us to rewrite Eq. (A1) as

$$
\begin{aligned}
P\left(\theta ; \bar{p}_{\|}, \bar{\theta}_{i}\right) \simeq & \frac{1}{4 \pi^{2} B_{1}\left(\bar{\theta}_{i}\right)} \int_{0}^{2 \pi} d q_{x} \int_{0}^{2 \pi} d q_{x}^{\prime} \\
& \times \exp \left(i \frac{\left(\theta+\bar{\theta}_{i}\right)\left(q-q^{\prime}\right)}{B_{1}\left(\bar{\theta}_{i}\right)}+\frac{i A_{x}}{\hbar}\left(\sin q-\sin q^{\prime}\right)\right. \\
& \left.-\frac{\sigma^{2}\left(q-q^{\prime}\right)^{2}}{4 B_{1}^{2}\left(\bar{\theta}_{i}\right)}\right) .
\end{aligned}
$$

Reverting to sum and difference variables as in Eq. (2.17) then gives

$$
\begin{aligned}
P\left(\theta ; \bar{p}_{\|}, \bar{\theta}_{i}\right) \simeq & \frac{1}{4 \pi^{2} B_{1}\left(\bar{\theta}_{i}\right)} \int_{0}^{2 \pi} d Q \int_{-Q}^{Q} d \Delta Q \\
& \times \exp \left(i \frac{\left(\theta+\bar{\theta}_{i}\right) \Delta Q}{B_{1}\left(\bar{\theta}_{i}\right)}+\frac{2 i A_{x}}{\hbar} \cos Q \sin \left(\frac{\Delta Q}{2}\right)\right. \\
& \left.-\frac{\sigma^{2} \Delta Q^{2}}{4 B_{1}^{2}\left(\bar{\theta}_{i}\right)}\right) .
\end{aligned}
$$

Scaling the difference variable as $\Delta r=\sigma \Delta Q$, noting that $\sigma$ is large so that the integration limits $\pm \sigma Q$ may be replaced with $\pm \infty$ and that $\sin (\Delta r /(2 \sigma)) \sim \Delta r /(2 \sigma)$ allows us to perform the Gaussian integral over the variable $\Delta r$,

$$
\begin{aligned}
P\left(\theta ; \bar{p}_{\|}, \bar{\theta}_{i}\right) \simeq & \frac{1}{\sqrt{\pi} \sigma 2 \pi} \int_{0}^{2 \pi} d Q \\
& \times \exp \left(-\frac{1}{\sigma^{2}}\left[\theta+\bar{\theta}_{i}+K \cos Q\right]^{2}\right),
\end{aligned}
$$

where we made use of the fact that $B_{1}\left(\bar{\theta}_{i}\right) A_{x} / \hbar=K$ (see Eqs. (2.25) and (2.32)). Changing variables such that

$$
\sin (K \cos Q)=\frac{K}{\sqrt{1+K^{2}}} \sin u
$$

and noting that to order $K^{2}$ one may assume that $\cos Q \simeq \sin u$ then gives the classical result as in Eq. (2.39).

${ }^{1}$ L. M. Hubbard and W. H. Miller, J. Chem. Phys. 78, 1801 (1983).

${ }^{2}$ R. R. Hancox, Phys. Rev. 42, 864 (1932).

${ }^{3}$ B. R. Williams, J. Chem. Phys. 55, 1315 (1971).

${ }^{4}$ G. Boato, P. Cantini, and L. Mattera, Surf. Sci. 55, 141 (1976).

${ }^{5}$ K. H. Rieder and W. Stocker, Phys. Rev. Lett. 52, 352 (1984).

${ }^{6}$ E. K. Schweizer and C. T. Rettner, Phys. Rev. Lett. 62, 3085 (1989).

${ }^{7}$ E. K. Schweizer, C. T. Rettner, and S. Holloway, Surf. Sci. 249, 335 (1991).

${ }^{8}$ T. Andersson, F. Althoff, P. Linde, M. Hassel, M. Persson, and S. Andersson, J. Chem. Phys. 113, 9262 (2000).

${ }^{9}$ T. Andersson, F. Althoff, P. Linde, S. Andersson, and K. Burke, Phys. Rev. B 65, 045409 (2002).

${ }^{10}$ A. Schueller, S. Wethekam, and H. Winter, Phys. Rev. Lett. 98, 016103 (2007).

${ }^{11}$ P. Rousseau, H. Khemliche, A. G. Borisov, and P. Roncin, Phys. Rev. Lett. 98, 016104 (2007).

${ }^{12}$ M. Arndt, O. Nairz, J. Voss-Andreae, C. Keller, G. van der Zouw, and A. Zeilinger, Nature (London) 401, 680 (1999).

${ }^{13}$ O. Nairz, M. Arndt, and A. Zeilinger, Am. J. Phys. 71, 319 (2003).

${ }^{14}$ M. Gring, S. Gerlich, S. Eibenberger, S. Nimmrichter, T. Berrada, M. Arndt, H. Ulbricht, K. Hornberger, M. Mueri, M. Mayor, M. Bockmann, and N. L. Doltsinis, Phys. Rev. A 81, 031604(R) (2010).

${ }^{15}$ J. M. Moix and E. Pollak, J. Chem. Phys. 134, 011103 (2011).

${ }^{16}$ W. H. Miller and F. T. Smith, Phys. Rev. A 17, 17 (1978).

${ }^{17}$ E. Pollak, S. Sengupta, and S. Miret-Artés, J. Chem. Phys. 129, 054107 (2008); E. Pollak and S. Miret-Artés, ibid. 130, 194710 (2009).

${ }^{18}$ E. Pollak, J. M. Moix, and S. Miret-Artés, Phys. Rev. B 80, 165420 (2009); Phys. Rev. Lett. 104, 116103 (2010).

${ }^{19}$ E. Pollak and S. Miret-Artés, Chem. Phys. 375, 337 (2010).

${ }^{20}$ E. Pollak and J. Tatchen, Phys. Rev. B 80, 115404 (2009).

${ }^{21}$ S. Miret-Artés and E. Pollak, Surf. Sci. Rep. 67, 161 (2012).

${ }^{22}$ T. Kondo, H. S. Kato, T. Yamada, S. Yamamoto, and M. Kawai, Eur. Phys. J. D 38, 129 (2006).

${ }^{23}$ L. M. Hubbard and W. H. Miller, J. Chem. Phys. 80, 5827 (1984).

${ }^{24}$ S. Daon, E. Pollak, and S. Miret-Artés, "Semiclassical perturbation theory for surface temperature effects in atom surface scattering" (unpublished). 